\title{
Erratum:
}

(Computational and Applied Mathematics, Vol. 22, N. 3, pp. 359-395)

\section{Steam injection into water-saturated porous rock}

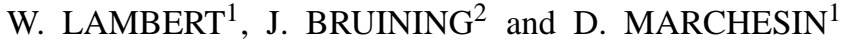 \\ ${ }^{1}$ Instituto Nacional de Matemática Pura e Aplicada, Estrada Dona Castorina 110, \\ 22460-320 Rio de Janeiro, RJ, Brazil \\ ${ }^{2}$ Dietz Laboratory, Centre of Technical Geoscience, Mijnbouwstraat 120, \\ 2628 RX Delft, The Netherlands \\ E-mails: lambert@fluid.impa.br / J.Bruining@mp.tudelft.nl / marchesi@impa.br
}

\begin{abstract}
The Riemann problem for steam injection at boiling temperature into a porous medium saturated with water was solved in [1]. Here, we correct the Riemann solution for case III and redraw the speed diagrams 3.1, 3.4 and 4.5. We redraw also the solution in case III, Figure 4.4.
\end{abstract}

Mathematical subject classification: 76S05, 35L60, 35L67.

Key words: Porous medium, steamdrive, Riemann solution, balance equations, multiphase flow.

\#558/02. Received: 28/XII/02. Accepted: 18/VIII/03.

This work was supported in part by: CNPq scholarship 141573/2002-3, ANP/PRH-32 scholarship, CNPq 301532/2003-6, CNPq 450161/2004-8, FAPERJ E-26/152.163/2002 and IM-AGIMB. 
In [1], cases I and II are correct, but there are some mistakes that influence the solution in case III. The first relevant mistake is the statement that the saturation $S_{\dagger}$ maximizes $v^{S C F}$ (Remark 11). The correct statement is:

Theorem 1. There are two saturation values that satisfy Eq. (3.7) in [1] for each fixed $T<T^{b}$. The smallest $S_{\dagger}$ minimizes $v^{S C F}$. The other $S_{\dagger \dagger}$ maximizes $v^{S C F}$, but it is irrelevant.

Moreover the solution is stable if we vary the left state, it follows that:

Corollary 1. In the limit as $S_{L}$ tends to zero, the wave speed $v_{g, w}^{b}$ in the hot region given by Eq. (2.8) in [1] converges to zero, so the solution for the Riemann problem reduces a cooling discontinuity in the liquid water region.

From the above Theorem and Corollary, we see that the Figures 3.1 and 3.4 in [1] contain an error; we correct them in Figure 1.
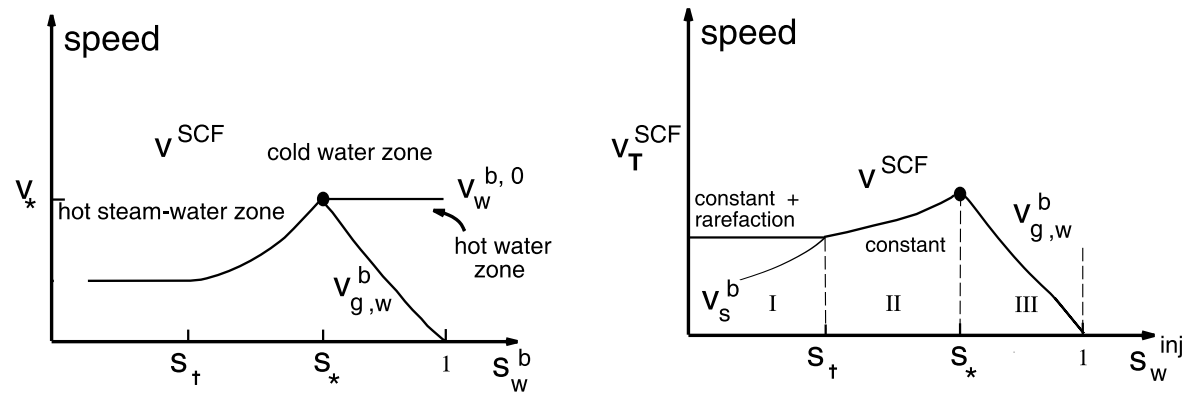

Figure $1-a$ )-Left: Corrected schematic bifurcation near $S^{*}$ (for fixed $u^{0}, T^{0}$ versus $S_{w}^{b}$. The shock speed $S C F$ is minimum at $S_{\dagger}$; the shock speed $v_{g, w}^{b}$ tends to zero when $S_{w}^{b}$ tends to 1. b)-Right: The corrected structure of steam-water zone below solid curve marked by $v_{s}^{b}, v_{T}^{S C F}, v^{S C F}$ given in Eqs. (2.7), (2.24) with $S_{w}=S_{\dagger}$, Eq. (2.24) with $S_{\dagger}<S^{i n j}<S_{*}$, and Eq. (2.8) respectively. The figures are not drawn to scale.

The wave speed diagram in Figure 4.5 of [1] contains an error also. The correct diagrams are given in Figure 2. There are two diagrams because the saturation shock speed and the thermal shock are different for injected saturations larger than $S_{*}$. These diagrams are drawn out of scale for illustrative purposes, because the characteristic speed $v_{s}^{g}$ can be much larger than the other wave speeds. Figs. 3.1 
and 3.4 in [1] contain an error also; those figures were summarized in Figure 4.5 in [1], which is corrected here in Figure 2. The Riemann solution for the cases I and II are correct, however the Riemann solution for case III contains an error. The mistake is the statement that the saturation shock speed $\lambda_{s}^{b}$ is faster than $v^{S C F}$ in cases I and II. The correct statement is that $v_{g, w}^{b}$ speed converges to zero if the water saturation at the left state tends to zero, as summarized in Corollary 1 above. The thermal wave speed is drawn in Figure 2.a) and in 2.b) we draw the saturation wave speed.
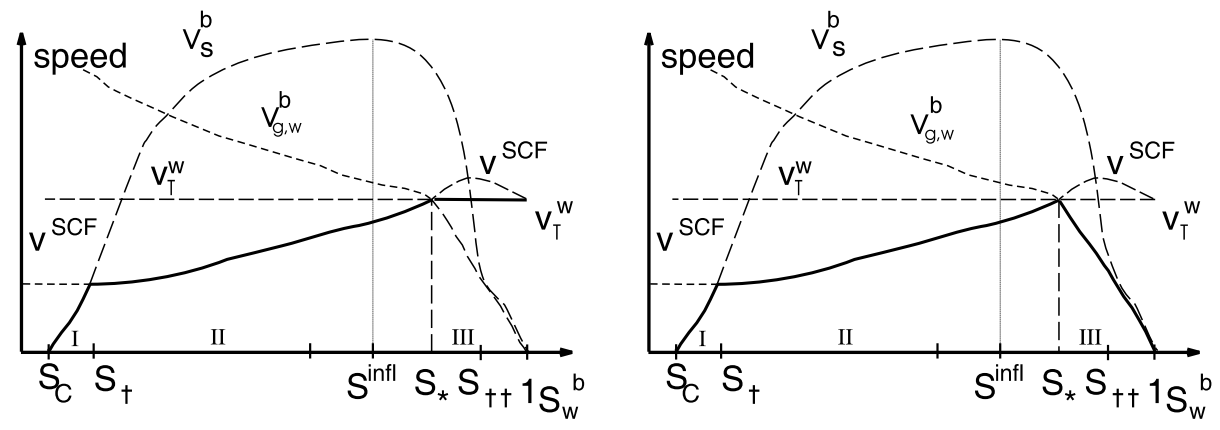

Figure $2-a$ )-Left: The diagram represents the thermal wave speed; $b$ )-Right: The diagram represents the saturation wave speed. They correct the diagram presented in Figure 4.5 of [1]. In both, the solid curve represents the wave speeds used in the Riemann solution. The characteristic speed $v_{s}^{b}$, Eq. (2.7), is the Buckley-Leverett characteristic speed in the hot region; the shock speed $v_{g, w}^{b}$ is the HISW speed, Eq. (2.8), and represents the Buckley-Leverett shock for a water saturation $S_{w}$ to $S_{w}=0 ; v^{S C F}$ is the condensation shock speed between the hot region and the liquid water region, Eq. (2.24); $v_{w}^{b, 0}$ is the cooling contact discontinuity speed in the liquid water region, Eq. (2.15). Notice that the temperature shock speed tends to $v_{T}^{W}$ when water saturation tends to $S_{w}=1$ (liquid water region) and the saturation shock speed tends to zero, thus the solution converges to the solution in the liquid water region.

The solution diagram for case III given in Figure 4.4 of [1] must be modified. The strength of saturation shock tends to zero when the injection saturation tends to 1 , while the speed of cooling discontinuity does not change. In Figure 3 we show the schematic solution for case III for three different injection saturations. 

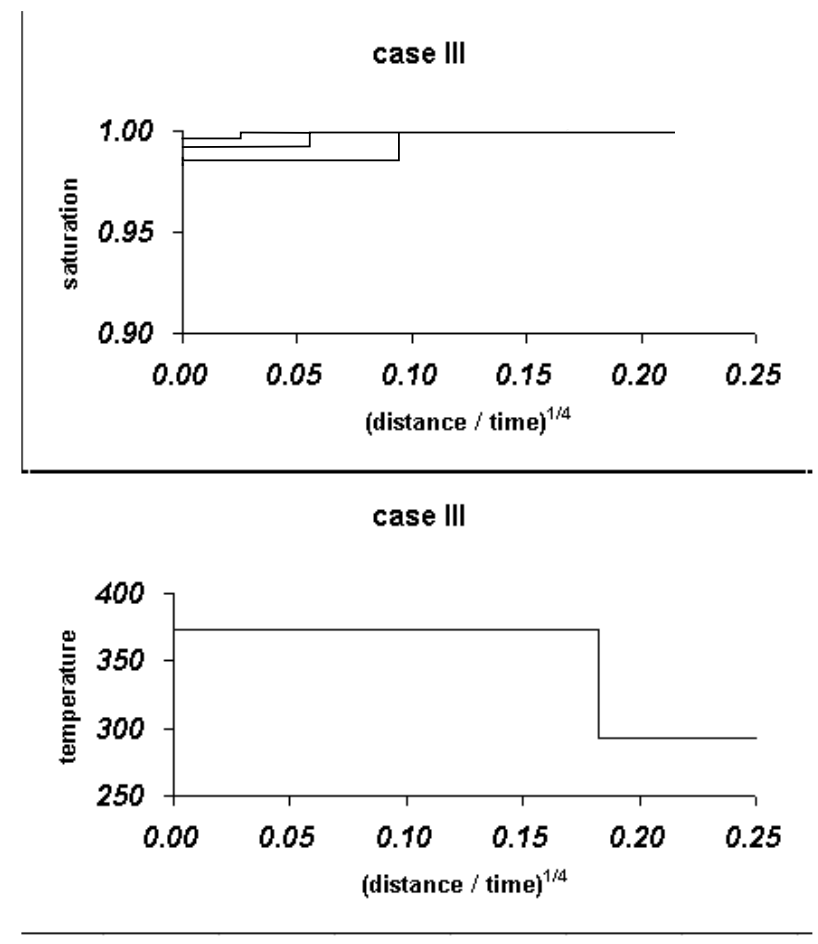

Figure 3 - Steam injection for three high water saturation values. We get a constant state upstream, the Buckley-Leverett saturation shock and the cooling discontinuity. These waves have distinct speeds; notice that $v_{g, w}^{b}$ tends to zero when the injection saturation tends to 1 , while the speed of cooling discontinuity does not change.

\section{REFERENCES}

[1] Bruining J., Marchesin D. and Van Duijn C.J. Steam Injection Into Water-Saturated Porous Rock. Computational and Applied Mathematics, 22(3) (2003), 359-395.

[2] Lambert W. Doctoral Thesis: IMPA, 2006, in preparation.

[3] Lambert W., Marchesin D. and Bruining J. The Riemann Solution of the balance equations for steam and water flow in a porous medium, submitted to Methods and Applications of Analysis 2005. 